\title{
NEURO-FUZZY EN EL MODELADO DEL COMPORTAMIENTO DE USUARIOS-TURÍSTICOS EN EWOM
}

\author{
R.M. Aguilar ${ }^{1}$, J.M. Torres ${ }^{2}$, J.A. Méndez ${ }^{3}$ y E. Parra-López ${ }^{4}$ \\ ${ }^{1,2,3}$ Dpto. Ingeniería Informática y de Sistemas, Universidad de La Laguna \\ ${ }^{4}$ Dpto. de Dirección de Empresas e Historia Económica \\ 38200 La Laguna (Tenerife), España. \\ 1'raguilar@ull.edu.es,,jmtorres@ull.edu.es, 3jamendez@ull.edu.es y ${ }^{4}$ eparra@ull.es
}

\section{Resumen}

La tecnología ha cambiado la forma de gestionar los viajes, la relación entre el viajero y las empresas del sector turístico y la forma en que los turistas comparten las experiencias del viaje. La transformación digital se impone para llegar a los nuevos perfiles de viajero, especialmente a los llamados usuarios-turistas de la Tercera Generación, nativos digitales que no entienden el mundo sin estar conectados y se aprovechan al 100\% de sus ventajas. En este contexto, las plataformas digitales donde los usuarios publican sus referencias sobre los servicios turísticos recibidos tienen cada vez más peso frente a los contenidos corporativos, creados por las empresas y marcas. Este trabajo tiene como objetivo comprender los patrones de comportamiento de los turistas cuando utilizan las plataformas y comparten las experiencias, así como conocer los efectos que estas plataformas tienen sobre la intención de compra por parte de los usuarios-turistas.

En este artículo se presenta el modelado del comportamiento del usuario, para determinar a partir de los comentarios expresados en una plataforma eWOM (boca a boca electrónico), la percepción que se tiene del servicio recibido y cómo se transmite. Para ello utilizamos una metodología neuro-fuzzy que nos permite el aprendizaje de este tipo de sistemas heurísticos.

Para obtener un modelo del comportamiento humano, se recurre a la lógica borrosa como herramienta matemática para formalizar la incertidumbre con la que los usuarios se expresan. El aprendizaje de este comportamiento borroso, se realiza a partir de multitud de casos obtenidos de plataformas eWOM, por lo tanto es de utilidad usar las redes neuronales artificiales que infieran los patrones que se presentan en esos datos.

Palabras Clave: lógica borrosa, eWOM, turismo, redes neuronales, big-data

\section{INTRODUCCIÓN}

El mundo está cambiando, no es una novedad. Cada vez se generan más datos y más rápido, los cuales, una vez transformados en información, aportan un valor diferencial al negocio. El problema es averiguar cómo se logran estos beneficios, cómo se accede a la pista de la generación de valor. Aunque no se puede capturar los datos procedentes del futuro, sí que es posible predecir lo que sucederá en base a datos del pasado, lo que se conoce como analítica predictiva. Por lo tanto se hace crucial para el crecimiento de la empresa elaborar pronósticos que optimicen las campañas de marketing y el comportamiento de la web corporativa para mejorar la respuesta de los usuarios.

Las puntuaciones predictivas que se obtienen de los modelos acerca de cada cliente informan de las acciones que deben adaptarse para lograr los objetivos: retener al cliente, venderle un producto de categoría superior o presentarle un nuevo servicio. Para hacer pronósticos sobre el comportamiento de los clientes, es necesario contar con información fiable y consistente. El trabajo que se presenta en este artículo, desarrolla esta línea y pretende crear modelos del comportamiento de los usuarios a partir de los comentarios que dejan del servicio recibido en las redes sociales.

Se denomina WOM (word-of-mouth) o "boca a boca" al proceso que llevan a cabo los usuarios al compartir información y opiniones sobre productos, servicios y marcas, de manera independiente a cualquier influencia comercial o empresarial. Cuando esta acción se realiza con ayuda de las Tecnologías de la Información y Comunicaciones (TIC) se le denomina eWOM o "boca a boca electrónico". Aunque el concepto es el mismo, por las propias características del eWOM hace que las implicaciones sean totalmente diferentes. Estas características se centran en la facilidad en la distribución del mensaje via los medios de comunicación sociales (social media en inglés), que son plataformas de comunicación en línea donde el contenido es creado por los propios usuarios mediante el uso de las tecnologías de la Web 2.0, que facilitan la edición, la 
publicación y el intercambio de información [1]. Podemos indicar que la incorporación del boca a boca en las redes sociales o web 2.0 tienen las siguientes propiedades:

- Gran capacidad de difusión, el usuario puede acceder a opiniones de personas desconocidas.

- Uso masivo por parte de usuarios de distintas edades y colectivos, compartiendo entre todos los diferentes puntos de vista.

- El mensaje se puede propagar rápidamente por varias vías: blogs, páginas web, redes sociales, grupos de mensajes simultáneos, $\ldots$

- Discusión multidireccional entre los usuarios que participan activamente con sus respuestas sobre la información presentada.

- Perdurabilidad en el tiempo, ya que las discusiones son subidas a la red para su referencia actual y futura.

- Credibilidad por ser información ofrecida por usuarios de forma espontánea y en principio, sin pretensiones comerciales.

Estos hechos hacen que la vigilancia de los eWOM por las empresas turísticas resulte de especial relevancia. Según [2] el eWOM es "cualquier declaración positiva o negativa hecha por potenciales clientes, actuales o ex acerca de un producto o empresa, que se pondrá a disposición de un gran número de personas e instituciones a través de internet". Por lo tanto, podemos inferir que el eWOM se compone de cinco elementos principales:

- Declaración: positiva, negativa o neutra (indiferencia)

- Comunicador (emisor): potencial cliente, actual o anterior

- Objeto: producto, servicio y/o empresa

- Receptor: multitud de personas e instituciones

- Entorno: internet, en particular los social media

En los casos de estudio en este trabajo tenemos que el objeto es el servicio recibido en los establecimientos hoteleros. La declaración serán el conjunto de comentarios realizados sobre el mismo. El emisor es el cliente que comenta su experiencia y los receptores son los posibles usuarios que buscan consejos sobre el servicio en la Red Social (entorno) y por lo tanto, queremos medir cómo influye en su intención de compra (Figura 1).

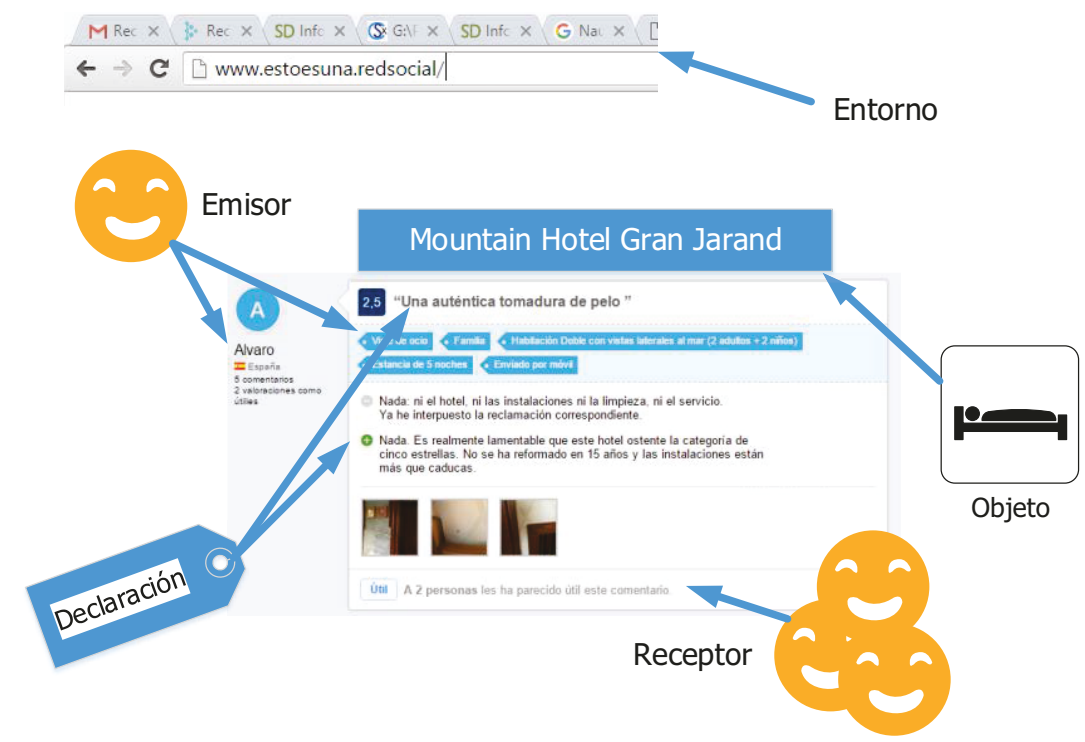

Figura 1: Elementos del eWOM

\section{EMISOR}

De acuerdo con Peppers y Rogers T.[3], "una empresa que se vuelca en sus clientes es una empresa que utiliza la información para obtener una ventaja competitiva y alcanzar el crecimiento y la rentabilidad. En su forma más generalizada Customer relationship management (CRM) puede ser considerado un conjunto de prácticas diseñadas, simplemente, para poner a una empresa en un contacto mucho más cercano con sus clientes. De este modo, aprender más acerca de cada uno, con el objetivo más amplio de que cada uno sea más valioso incrementando el valor de la empresa." Es por ello 
que cuando el modelo de gestión lo basamos en la satisfacción del cliente debemos trabajar en 4 dimensiones: Identificación del cliente, Atracción del cliente, Retención del cliente y Customer Development. Esta cuarta dimensión habla de descubrir el verdadero mercado para tu empresa y producto, apostando por descubrir y aprender de los propios clientes. Focalizarnos en el cliente nos permite desarrollar el producto que necesita de verdad el usuario. En definitiva, lo que se quiere es tener un conocimiento profundo del cliente. En este trabajo, se quiere realizar un modelo del usuario de establecimiento hotelero, que nos permita predecir su comportamiento en las redes sociales, y por lo tanto la implicación que tendrá en la intención de compra de los receptores de dichos comentarios. Esto es, se pretende tener un modelo que indique cómo se expresará el cliente una vez recibido su servicio, en un entorno eWOM. Para ello vamos a hacer uso del dataminig, ya que el comportamiento se extraerá de la multitud de comentarios dejados en las plataformas por los usuarios turísticos.

El objetivo es construir el modelo que describa cómo publicará su percepción el usuario de los establecimientos turísticos. El intercambio de información entre emisor y receptor, sobre el objeto en cuestión (establecimientos hoteleros) se realiza a través de declaraciones realizadas en entornos TI eficientes y fáciles de usar (redes sociales) que promueven este intercambio de información. Por lo tanto, el entorno tecnológico utilizado no resulta una barrera para la adecuada comunicación. Si bien la información se recoge, difunde y comparte por todo el mundo a través de internet fácilmente, la calidad de la información no se asegura. Se evidencia que la calidad de la información es uno de los aspectos más importantes del uso de la información disponible en Internet. Naumann (2001)[4] ha señalado el papel significativo de la calidad de la información de una nueva era digital mediante el axioma : "la calidad de la información es el tiempo de respuesta de la era de Internet". En este trabajo, se explora el papel crítico de intercambio de información en el vínculo entre la calidad de la información y rendimiento de la misma en la intención de compra del receptor de la declaración.

La baja calidad de la información es uno de los problemas más acuciantes para consumidores de información que se distribuye por fuentes autónomas. Esto es cierto para todo el rango de los usuarios de servicios de información vía redes sociales. La necesidad de adoptar medidas contra la baja calidad es clara y se han propuesto muchos métodos para mejorarla. Sin embargo, la mayoría de los enfoques tienen carencias sobre cómo evaluar las puntuaciones de calidad en primer lugar [5]. Evaluar un índice de calidad es complicado por las siguientes razones:
- Los criterios de calidad son a menudo de naturaleza subjetiva, por tanto, no es posible evaluar de forma automática, es decir, independiente del usuario.

- Las fuentes de información por lo general son autónomas y no siguen un patrón a la hora de comunicar.

- La enorme cantidad de datos a ser evaluados impide la evaluación de toda la información de manera conjunta.

- La información de fuentes autónomas está sujeto a cambios, a veces sorprendentes en el contenido y la calidad.

Se han realizado muchos intentos para recopilar y clasificar los criterios de calidad de información. En este trabajo utilizamos los criterios establecidos por Naumann (2000)[6] que indica que la Calidad de la información está influenciada por tres factores principales: la percepción del usuario, la información sí, y el proceso de acceso a la información. Para el caso que nos ocupa, el estudio de los eWOM turísticos nos centramos en modelar la calidad respecto a la percepción del usuario, estudiando los parámetros de relevancia, precisión, sesgo y usabilidad en los comentarios publicados por los usuarios de alojamientos turísticos.

Por ello, se hace necesario estudiar los componentes que hacen que los comentarios en una plataforma tengan más calidad y por lo tanto, incremente la intención de compra en los receptores. Para ello se estudia lo expresado por los usuarios sobre su estancia en hoteles. Los elementos a tener en cuenta, en cualquier comunicación, y por lo tanto en el eWOM son:

- Relevancia: el grado de importancia de acuerdo a una necesidad concreta de información.

- Precisión: la cantidad de datos y hechos que pueden ser contrastados con otras fuentes.

- Sesgo: el peso que tiene el punto de vista individual del autor.

- Usabilidad: todo lo que debemos conocer para acceder al servicio en las mejores condiciones posibles

El modelado de estos elementos resulta complejo ya que recogen las incertidumbres del comportamiento del usuario que viene especificado de manera vaga o ambigua. Por ello hay que recurrir a la teoría de conjuntos borrosos. Mediante la definición de variables lingüísticas (precisión, relevancia, usabilidad, sesgo) que adoptan valores también limgüisticos (alta, media, buena, baja, mala), y mediante el establecimiento de las normas sobre la base de dichas variables, se puede modelar dichos comportamientos imprecisos [7][8][9] (Figura 2). Las ventajas del modelado borroso son dos. En primer 
lugar, el ofrece un nuevo mecanismo para la aplicación de las leyes de comportamiento que a menudo se basan en el conocimiento o en las descripciones lingüísticas. En segundo lugar, proporciona una metodología alternativa para facilitar el diseño de regresiones no lineales para los sistemas que se basan en comportamiento incierto que es muy difícil de relacionar con la teoría convencional de modelos no lineales [10][11].

\subsection{REGRESIÓN BORROSA PARA MODELAR AL EMISOR EWOM}

Al no existir un procedimiento sistemático generalizado para el diseño de sistemas de lógica borrosa en turismo, la aplicación a una nueva área de conocimiento de este tipo de sistemas se basa en el análisis de ejemplos [12].

Los sistemas de inferencia borrosa constituyen una clase de algoritmos de cómputo basados en conjuntos difusos y en el razonamiento aproximado. Se componen de tres elementos (Figura 2). En primer lugar un conjunto de reglas lógicas ("Si precisión=alta entonces intención_compra=alta), con múltiples antecedentes y un consecuente, denominado base de reglas. En segundo lugar el llamado diccionario que contiene la definición de los conjuntos difusos asociados a los antecedentes y consecuentes de las reglas (precisión, relevancia, sesgo, usabilidad, intención compra). Por último, hay que definir un mecanismo de inferencia.

El sistema de lógica difusa que da respuesta al objetivo planteado se muestra en la figura 3. El subsistemal vincula, a través de un conjunto de reglas, una serie de entradas (relevancia, precisión, sesgo y usabilidad de la información) para el sistema borroso que se va a inferir con el conocimiento del experto, de manera que se obtiene la calidad de la información percibida por el usuario. El subsistema2 infiere del conocimiento del experto la fiabilidad de la información percibida por el usuario, a partir de las dos entradas (fiabilidad fuente y fiabilidad de la plataforma).

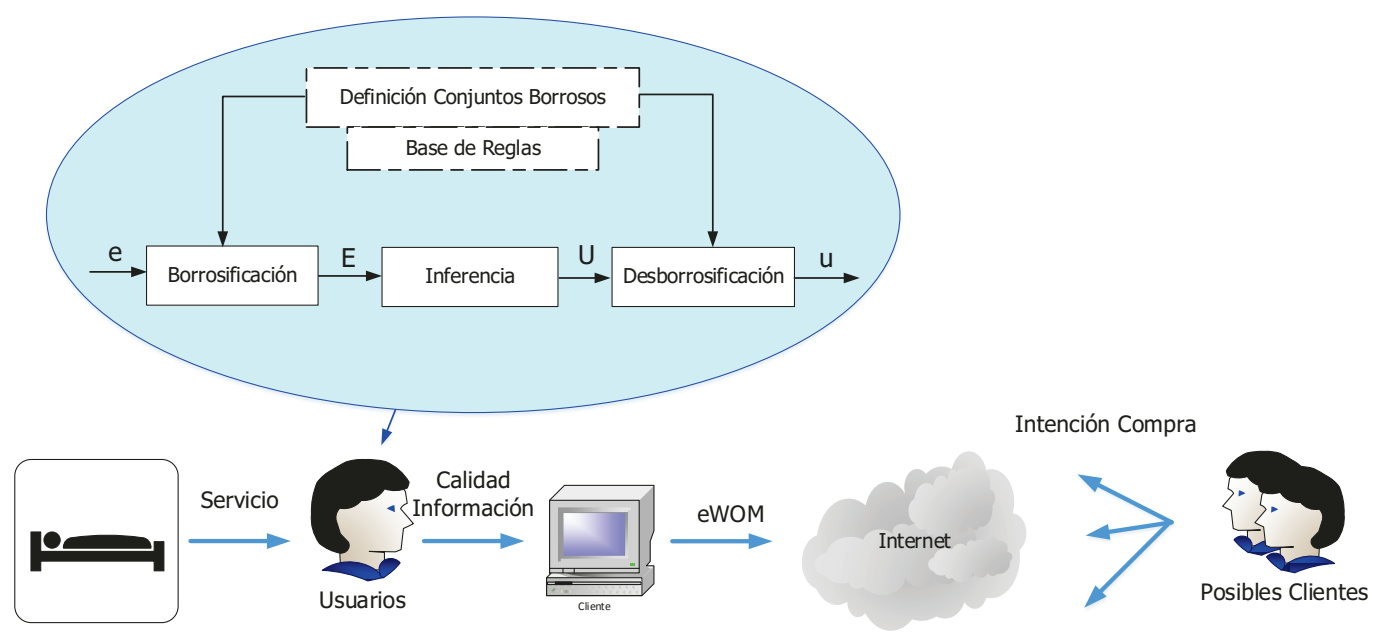

Figura 2. Sistema de inferencia borrosa para el modelado del comportamiento del usuario turístico.

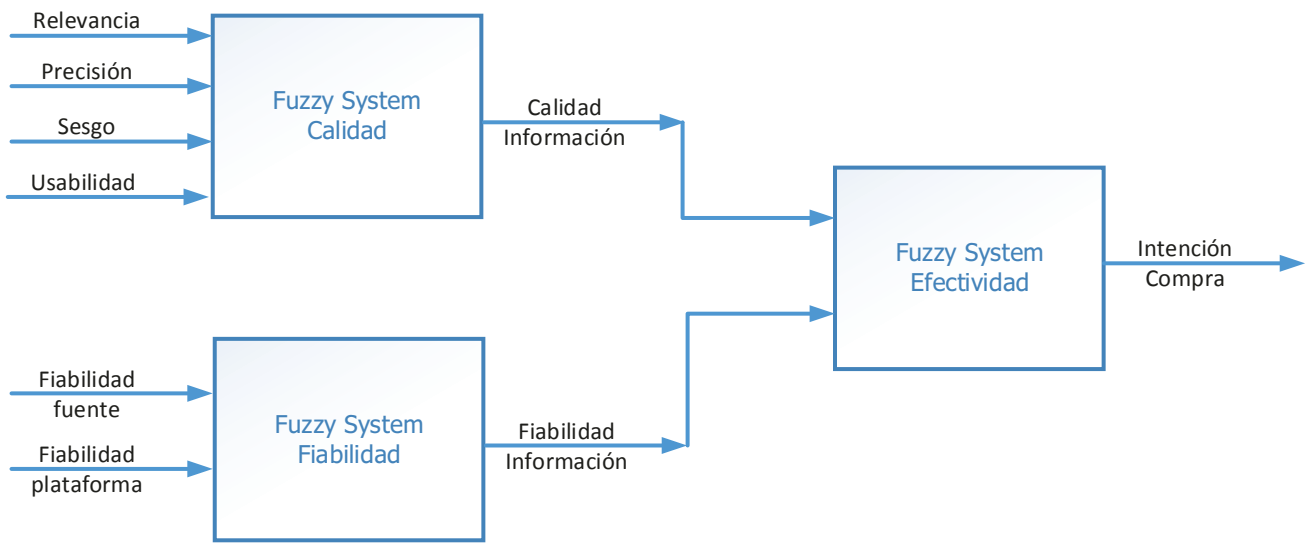

Figura 3 Diagrama de bloques del sistema de inferencia difusa para la determinación de la intención de compra de productos turísticos a partir de eWOM. 
Finalmente, se define un tercer bloque que establece la relación entre la calidad y la fiabilidad de la información para determinar la intención de compra del servicio por parte del usuario. En el proceso de inferencia se usan los valores lingüísticos de las entradas y el conjunto de reglas, definidas a partir del conocimiento de los expertos, para obtener una salida con un valor lingüístico (figura 3 ). El proceso final consiste en la desborrosificación, donde se convierte el valor lingüístico de la salida en un valor conciso o crisp.

Para cada uno de los sistemas borrosos, hay que definir los conjuntos borrosos que describen las variables linguisticas (relevancia, precisión, sesgo, usabilidad, calidad, fiabilidad e intención de compra) según son manejadas por el usuario. Asimismo, hay que determinar la relación (causa-efecto) entre las variables de entrada y las de salida. La formalización de ese conocimiento se realiza a partir del conocimiento heurístico de los usuarios.

Para definir las entradas, salidas y reglas del sistema es necesario partir de la base de conocimiento heurístico de los usuarios de plataformas turísticas, respecto a su comportamiento relacionado con la intención de compra de servicios turísticos según lo comentado en esas plataformas. Para ello se utilizan los comentarios publicados en las plataformas, como los que se observan en la figura 4. A partir de estas declaraciones, se extrae el valor lingüístico para cada una de las variables de entrada: relevancia (bajo, medio, alto), precisión (bajo, medio, alto, muy alto), interpretability (bajo, medio, alto) y value-added (bajo, medio, alto, muy-alto).

El siguiente paso es definir el conjunto de reglas de tipo "Si antecedente entonces consecuente" que con los valores de entrada nos permita inferir el valor lingüístico de la variable de salida: calidad_información (baja, media, alta). Debido al número de combinaciones posibles de valores de entrada con los valores de salida, es necesario recurrir a un método de data-mining que permita extraer este modelo de los datos de forma automática. Para ello se recurre al uso de Redes Neuronales Artificiales.

Utilizamos un algoritmo neuro-fuzzy consistente en una red neuronal que extrae la relación que existe entre los datos borrosos de la entrada y el valor conciso que el emisor indica como valoración del servicio. Esta combinación de lógica borrosa y RN nos permite obtener un modelo sobre la calidad de la información que publica el usuario en las redes. La salida de este modelo combinado con los valores de fiabilidad de la plataforma y el usuario, nos determinan la intención de comprar de los usuarios receptores.

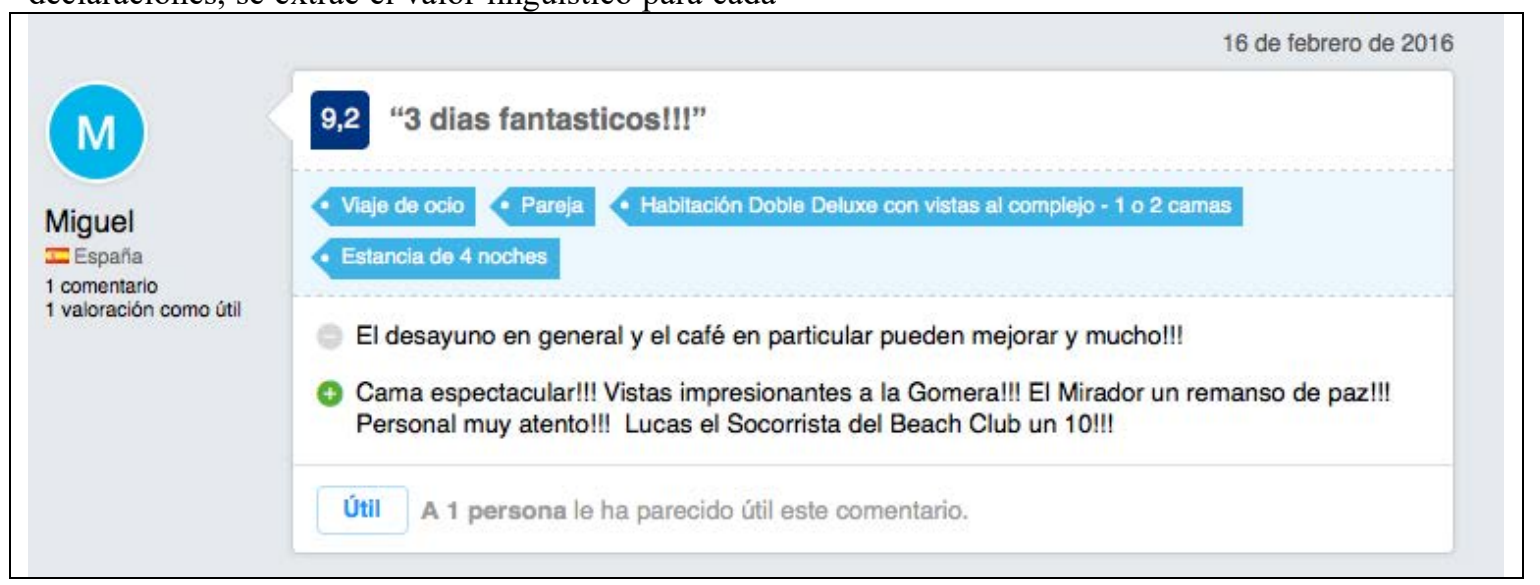

Figura 4 Comentarios sobre servicio en establecimientos hoteleros

\subsection{MODELO NEURO-BORROSO}

Debido a las características del conocimiento impreciso a modelar, se ha implementado un sistema neuroborroso para determinar la calidad de la información que publicará el usuario turístico. Se propone un diseño, como el que se muestra en la figura 3 , en el que conocido la relevancia, precisión, sesgo y usabilidad de la información en la web, el simulador nuero-borroso determinará el nivel de calidad de la información que se presenta a los usuarios potenciales.

El uso de lógica borrosa en este modelado tiene una aplicación inmediata debido a que su comportamiento se conoce en base a reglas definidas de forma poco precisa. Esta imprecisión surge de la complejidad del propio sistema. La manera de atacar este problema es reducir la complejidad aumentando la incertidumbre sobre las variables. El comportamiento del simulador del emisor viene dado 
por una serie de reglas que utilizan términos lingüísticos cargados de incertidumbre. Así se formulan reglas del tipo "SI Relevancia es alta y Precision es Alta ENTONCES la Calidad es alta". A este tipo de modelo borroso se le conoce como Mandani; pero en problemas complejos y de alta dimensionalidad (como el que nos ocupa) requiere un número de reglas elevado. Para solventar este incoveniente utilizaeremos el modeldo borroso de Takagi, Sugeno, y Kang (TSK) [13] que sustituye la variable de salida por una función matemática en el consecuente, de forma que el formato general de regla en inferencia TSK es:

"SI relevancia es Alta y Precision es Alta ENTONCES Calidad es f(Relevancia, Precision)”.

Por tanto, se dispone de una base de conocimiento del sistema, esto es, un conjunto de reglas que tratan de modelar el resultado en el eWOM a partir de los comentarios presentados. Los sistemas de reglas difusas se basaban en la información suministrada por expertos; sin embargo, para el caso de sistemas complejos como el que nos ocupa, las reglas así construidas no permitían una simulación aceptable del eWOM. La búsqueda de sistemas difusos que aproximen de manera aceptable la dinámica de sistemas complejos ha conllevado al desarrollo de técnicas de extracción de reglas difusas a partir de datos de entrada y salida. En este trabajo se propone el uso de Redes Neuronales para la identificación de las reglas borrosas. Dichas reglas se infieren a partir de una red neuronal, que es capaz de determinar las relaciones existentes entre el conjunto de datos de entradas (relevancia, precisión, sesgo, usabilidad) con el dato de salida (calidad de información).

El sistema neuro-borroso de predicción de la calidad de la información está formado por tres elementos:

- Conjunto de reglas lógicas, con múltiples antecedentes y un consecuente, denominado base de reglas. Se utilizaran el modelo TSK.
- Diccionario que contiene la definición de los conjuntos borrosos asociados a los antecedentes de las reglas

- Mecanismo de inferencia.

\subsection{1.- Diccionario del sistema neuro-borroso}

$\mathrm{Si}$ consideramos los criterios establecidos por Naumann (2000) [6] que indica que la Calidad de la información nuestro diccionario estará formado por 4 variables de entrada (relevancia - R, precisión - P, sesgo - S y usabilidad - U). En la figura 5 se muestra la descripción lingüística de la variable Relevancia. La salida del sistema será calidad de la información y nos vendrá dada por una función de las variables de entrada. Por lo general esta función es un polinomio en las variables de entrada. Esto es:

$$
\text { Calidad_Información }=f(\mathrm{R}, \mathrm{P}, \mathrm{S}, \mathrm{U})
$$

\subsection{2.- Base de reglas y mecanismo de inferencia}

Los modelos borrosos usan reglas "IFTHEN" para establecer relaciones cualitativas entre las variables. La naturaleza basada en reglas de los modelos borrosos permite el uso de información basada en forma de sentencias de lenguaje natural y en consecuencia, hacen los modelos más transparentes al análisis y a la interpretación. Las cuantificaciones lingüísticas, definidas anteriormente, se utilizan para especificar un conjunto de reglas que capturen el comportamiento del emisor del eWOM acerca de la calidad de información que suministra a los receptores. Por ejemplo, tendremos la siguiente regla:

SI 'Relevancia' es Muy_Baja y 'Precisión' es Poca y 'Sesgo' es Sin_sesgo y 'Usabilidad' es Nada ENTONCES 'Calidad_Información’ es Baja

\begin{tabular}{|l|l|l|}
\hline $\begin{array}{l}\text { Variable } \\
\text { entrada }\end{array}$ & $\begin{array}{l}\text { La relevancia de la información habla de que un tema recibe una cobertura } \\
\text { significativa }\end{array}$ \\
\hline $\begin{array}{l}\text { Números } \\
\text { borrosos }\end{array}$ & &
\end{tabular}

Figura 5 Variable de entrada relevancia

En lugar de trabajar con una salida borrosa, Takagi, Sugeno y Kang [14] (TSK) propusieron un nuevo modelo basado en reglas donde el antecedente está compuesto de variables lingüísticas y el consecuente se representa como una función lineal de las variables de entrada. El cálculo del lado izquierdo de 
las reglas borrosas en estos sistemas consiste en aplicar el operador de implicación escogido obteniendo un grado de pertenencia o activación para cada una de las reglas disparadas. En el lado derecho de estas reglas se obtiene el respectivo valor de salida mediante la combinación lineal de las entradas: $\mathrm{v}_{\mathrm{j}}=\mathrm{f}$ $\left(\mathrm{u}_{1}, \mathrm{u}_{2}, \ldots \mathrm{u}_{\mathrm{n}}\right)$ donde el subíndice en la variable de salida $\mathrm{V}_{\mathrm{j}}$ se refiere al número de la regla disparada. Este es el modelo a utilizar porque permite la obtención del sistema borroso a partir de las entradas cualitativas y la salida cuantitativa existente en todos los comentarios web (Figura 6).

La determinación de todas las reglas que especifican el modelo del emisor eWOM resulta una tarea engorrosa debido a la incertidumbre que se produce en dicha simulación. Una forma de aprender dichas reglas, a partir de los comentarios publicados en la web, es utilizar las redes neuronales que como se ha indica anteriormente son capaces de determinar las relaciones entre los datos de entrada/salida. Las redes neuronales, como paradigmas o modelos matemáticos simplificados de modelos semejantes al cerebro humano, funcionan como redes de computación de distribución paralela. Quizás la ventaja más importante de las redes neuronales es su adaptabilidad. Ellas pueden automáticamente ajustar sus parámetros (pesos) para optimizar comportamiento. La adaptación permite que puedan funcionar correctamente, aun cuando el ambiente o el sistema controlado varíe en el tiempo. Hay muchos problemas de identificación que pueden beneficiarse con una adaptación y modelación no lineal continua. Mientras la lógica borrosa ejecuta un mecanismo de inferencia bajo conocimiento impreciso, las redes

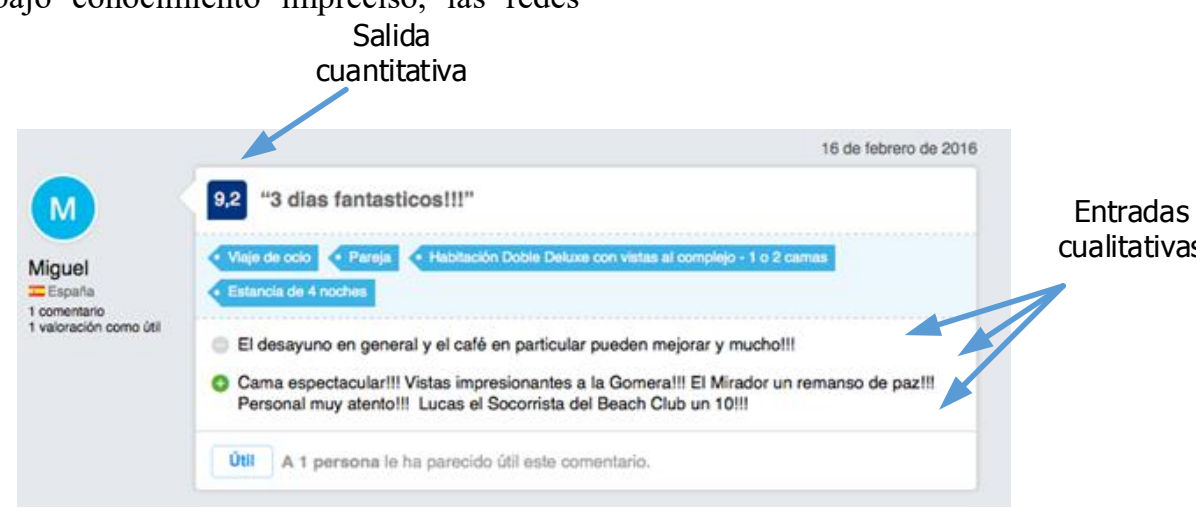

Figura 6 Datos cualitativos y cuantitativos en un comentario eWOM

Para la obtención del modelo TSK para el modelado del emisor turístico se obtuvieron los datos de entrada salida del proceso formado por el vector: [relevancia, precisión, sesgo, usabilidad] y como salida el vector de datos de cuantificación de grado satisfacción. A partir de los mismos se elaboran los conjuntos de datos de entrenamiento y de chequeo del modelo del sistema de inferencia borroso. Una vez entrado el sistema se obtuvo el modelo que infiere la calidad de la información a partir de los neuronales ofrecen ventajas tales como: aprendizaje, adaptación, tolerancia al fallo, paralelismo y generalización. Aunque la lógica borrosa puede codificar el conocimiento del experto directamente, usando reglas con etiquetas lingüísticas, usualmente toma una gran cantidad de tiempo diseñar $\mathrm{y}$ sintonizar las funciones de pertenencia que definen cuantitativamente dichas etiquetas lingüísticas. Las técnicas de aprendizaje de las redes neuronales permiten automatizar este proceso y reducir sustancialmente el tiempo de desarrollo y el costo mientras que se mejora el rendimiento. De la combinación de ambas técnicas resultan los sistemas híbridos llamados sistemas neuro-difusos

En este trabajo se propone el uso de la arquitectura ANFIS (Adaptive Neural Fuzzy Inference Systems) (Jang, 1993) para el ajuste de los parámetros del sistema borroso de modelado del emisor eWOM, a partir de pares de datos de entrada borrosa vs salida cuantitativa. Funcionalmente equivalente a un sistema borroso, el proceso de inferencia se implementa como una red neuronal que se ajusta por medio de técnicas de gradiente descenderte y mínimos cuadrados, y es capaz de ajustar los parámetros del antecedente $\mathrm{y}$ del consecuente. ANFIS es un sistema neuro-difuso que combina las ventajas de los sistemas borrosos y de las redes neuronales. Como sistema difuso no requiere de un gran conjunto de datos y proporciona transparencia, suavidad y representación del conocimiento previo. Como una red neuronal, proporciona adaptabilidad paramétrica

valores lingüísticos de las entradas. El ajuste se ha realizado con 100 muestras de entrenamiento, y se ha testeado con 20 muestras obteniendo un Error Cuadrático Medio $=1.8$.

\section{CONCLUSIONES}

Aunque se trate de un concepto relativamente nuevo el eWOM deberá ser tenido en cuenta por los promotores de productos y servicios turísticos dado 
el crecimiento que está teniendo éste en la Red. No cabe duda que la importancia de la reputación online de los productos y servicios turísticos, dada la tendencia actual de reservar a través de la Red y de hacer uso de la misma por parte de los viajeros prospectivos para informarse sobre las mejores opciones, modifica por completo las prácticas tanto de los usuarios como de los comercializadores. En ese sentido la Social Media representa una de las mayores innovaciones en materia de marketing online dentro del sector turístico de las aparecidas hasta el momento y susceptibles, dada su constante evolución, de importantes oportunidades de investigación.

El objetivo es acercar el producto a las necesidades del usuario, para lo que se necesita determinar a partir de los comentarios de las redes sociales el comportamiento de futuros turistas. El modelado de tal sistema resulta fundamental para comprender las interacciones producidas en un entorno eWOM.

Para conocer la forma de proceder de estas nuevas formas de comunicar, se hace esencial disponer de modelos que reflejen el comportamiento tanto de usuarios como posibles usuarios ante los comentarios aparecidos en las redes sociales. El uso combinado de la lógica borrosa y las redes neuronales nos permite modelar el comportamiento impreciso de los emisores de comentarios turísticos, para determinar el grado de influencia en el proceso de toma de decisión de los viajeros prospectivos que hacen uso del eWOM.

En este artículo se presenta el modelado del emisor eWOM utilizando como datos para la determinación de los patrones de comportamiento los comentarios existentes en las redes sociales. El proceso de inferencia resultante es una tarea costosa debida a la multitud de posibles opciones detectadas, por ello se hizo fundamental el uso de redes neuronales que determinaron las relaciones entre información cuantitativa y cualidativa de los comentarios eWOM. Quedan líneas abiertas como la determinación automática de los sentimientos a partir de los textos, o la determinación de acciones de control que minimicen influencias no deseadas.

\section{Referencias}

[1] Andreas M. Kaplan, Michael Haenlein, Users of the world, unite! The challenges and opportunities of Social Media, Business Horizons, Volume 53, Issue 1, JanuaryFebruary 2010, Pages 59-68, ISSN 0007-6813

[2] Thorsten Hennig-Thurau, Kevin P. Gwinner, Gianfranco Walsh, Dwayne D. Gremler, Electronic word-of-mouth via consumeropinion platforms: What motivates consumers to articulate themselves on the Internet?, Journal of Interactive Marketing, Volume 18, Issue 1, 2004, Pages 38-52, ISSN 1094-9968

[3] Peppers D. y Rogers M. (2011), Managing Customer Relationships: A Strategic Framework (Second Edition), Wiley.

[4] Naumann, F. (2001). From Databases to Information Systems - Information Quality Makes the Difference. In Proceedings of the 6th International Conference on Information Quality, (pp 244-260). MIT Sloan School of Management.

[5] C. Marinagi, P. Trivellas, P. Reklitis, Information Quality and Supply Chain Performance: The Mediating Role of Information Sharing, Procedia - Social and Behavioral Sciences, Volume 175, 12 February 2015, Pages 473-479, ISSN 1877-0428

[6] Naumann, F., \& Rolker, C. (2000). Assessment Methods for Information Quality Criteria. In Proceedings of the International Conference on Information Quality. In Proceedings of the 5th International Conference on Information Quality.(pp 148-162), Boston, USA.

[7] Chen G. \& Joo Y. H. (2009). Introduction to Fuzzy Control Systems, In: Encyclopedia of Artificial Intelligence, J. R. Rabul, J. Dorado, and A. Pazos (Eds.), 688-695, Hersh, ISBN 9781599048499, La Coruña, España

[8] Pedrycz W. (1993). Fuzzy Control and Fuzzy Systems, Research Studies. Press/John Wiley, ISBN O-471-93475-5, Taunton, New York

[9] Babuška R. (1998). Fuzzy Modeling for Control, Kluwer Academic Publishers, ISBN 978-0- 7923-8154-9, Boston, USA

[10] Li H. X. \& Tong S.C. (2003). A hybrid adaptive fuzzy control for a class of nonlinear mimo systems. IEEE Trans. Fuzzy Systems, Vol.11, No.1, (February 2003), pp. 24-34, ISSN 10636706

[11] Sala A., Guerra T. M. \& Babuška R. (2005). Perspectives of Fuzzy Systems and Control. Fuzzy Sets and Systems, Vol.156, No.3, (December 2005), pp. 432-444, ISSN 01650114, North-Holland

[12] Passino K, Yurkovich S. Fuzzy Control 2010;20106837:55-1-55-27.

[13] T. Takagi, M. Sugeno, Fuzzy identification of systems and its applications to modeling and control, IEEE Trans. Syst., Man, Cybern., 15 (1) (1985), pp. 116-132

[14] J.Jang, C. Sun, and, E. Mizutani, Neuro-fuzzy and Soft Computing, Prentice Hall 1997 